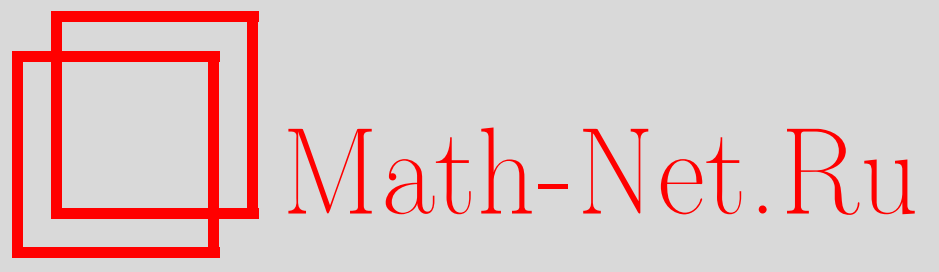

O. E. Barndorff-Nielsen, J. Schmiegel, A stochastic differential equation framework for the timewise dynamics of turbulent velocities, Теория вероятн. и ее примен., 2007, том 52, выпуск 3, 541-561

DOI: https://doi.org/10.4213/tvp77

Использование Общероссийского математического портала Math-Net.Ru подразумевает, что вы прочитали и согласны с пользовательским соглашением

http: //www . mathnet.ru/rus/agreement

Параметры загрузки:

IP : 3.81 .55 .215

26 апреля 2023 г., 17:04:45

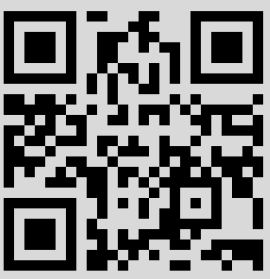


(C) $2007 \Gamma$.

BARNDORFF-NIELSEN O. E.*, SCHMIEGEL J.

\title{
A STOCHASTIC DIFFERENTIAL EQUATION FRAMEWORK FOR THE TIMEWISE DYNAMICS OF TURBULENT VELOCITIES
}

\begin{abstract}
В статье обсуждается некоторое стохастическое дифференциальное уравнение как модель динамики во времени турбулентных скоростей. Это уравнение способно «ухватывать» основные факты статистики временнь́х приращений скоростей. В частности, мы сосредотачиваем внимание на эволюции плотности вероятностей для приращений скоростей, имеющей нормальную обратную гауссовскую форму с тяжелыми хвостами при малых масштабах и приблизительно гауссовскими хвостами при больших масштабах. Кроме того, мы показываем, что предлагаемая модель согласуется с данными экспериментальной проверки уточненных гипотез подобия Колмогорова.
\end{abstract}

Ключевые слова и фразы: рассеяние энергии, перемежаемость, обратное гауссовское распределение, нормальное обратное гауссовское распределение, уточненные гипотезы подобия, турбулентность.

1. Introduction. The present paper proposes a class of stochastic differential equations aimed at modelling the timewise development of the main (longitudinal) component of the velocity vector, at a single location, in a turbulent fluid field. It represents a step in a project to formulate a full-fledged tempo-spatial stochastic process model for the three-dimensional velocity field. Previous parts of this project are discussed in [1] and [2]. The full model should ideally accord with the theory of homogeneous and isotropic turbulence, due to Kolmogorov and Obukhov, and with further key phenomenological features, stylized from detailed studies of major empirical data sets, such as those discussed in [1].

In order to make the considerations in the paper more directly accessible for physicists, our style of writing deviates somewhat from the traditional mathematical style of «Theory of Probability and Its Applications».

We stress that the model type presented here is only for the timewise dynamics, at a single location, of the velocity component in the mean flow

* Thiele Centre for Applied Mathematics in Natural Science, Department of Mathematical Sciences, University of Aarhus, DK-8000 Aarhus, Denmark; e-mail: oebn@imf.au.dk, schmiegl@imf.au.dk 
direction. However, most of the reliable and extensive data sets available are precisely for this component and, in fact, as concerns measurements of spatial variations very little empirical evidence exists, due to the difficulty of measuring this; see, however, [25] and [21].

On the other hand, the Kolmogorov hypotheses are formulated in terms of the spatial variations. To connect to the timewise regime the generally adopted approach is to translate results from the spatial setting to the temporal via Taylor's Frozen Flow Hypothesis, as will be described in Section 4.1.

It is also important to underline that Kolmogorov's theory concerns the asymptotic behavior as the Reynolds number tends to infinity. However, with the ample empirical evidence presently available it is clear that even for very large, but realistic, Reynolds numbers the hypothesized asymptotic regime has not been reached. Thus Kolmogorov's hypotheses give only a rough guide to the statistical properties of the velocities, and it is pertinent to seek extensions of his results that can cover a much wider spectrum of Reynolds numbers and timewise or spatial ranges. In fact, a universality result of the kind in question was established in [1] and is briefly described in Section 2 below.

The outline of the paper is as follows. Section 2 provides some background on the phenomenology of turbulent flows. In Section 3 we present our stochastic framework for the timewise dynamics of turbulent velocities. Section 4 indicates the necessary mathematical background on quadratic variation, (Brownian) semimartingales, infinitely divisible distributions and Lévy processes, which are the main building blocks of the model. Quadratic variation is proposed as a natural substitute for the usual definition of the integrated energy dissipation, which is not applicable for nondifferentiable stochastic processes. Section 5 discusses the timewise dynamics of the model for the turbulent velocity. The focus is on the evolution of the probability density function (PDF) of velocity increments across scales and on statistics related to Kolmogorov's refined similarity hypotheses. The theoretical results are illustrated and supplemented in Section 6 through simulations. Section 7 concludes with an outlook.

2. Phenomenological background. Since the pioneering work of Kolmogorov [13] and Obukhov [16], intermittency of the turbulent velocity field is of major interest in turbulence research. From a probabilistic point of view, intermittency refers, in particular, to the increase of the non-Gaussian behavior of the PDF of velocity increments with decreasing scale. A typical scenario is characterized by an approximately Gaussian shape for the large scales (larger than scales at the so-called inertial range), turning to exponential tails within the inertial range and stretched exponential tails for dissipation scales (below the inertial range) [7], [26].

It was reported in [1] that the evolution of the PDF of velocity increments for all amplitudes and all scales can be described within one class of 

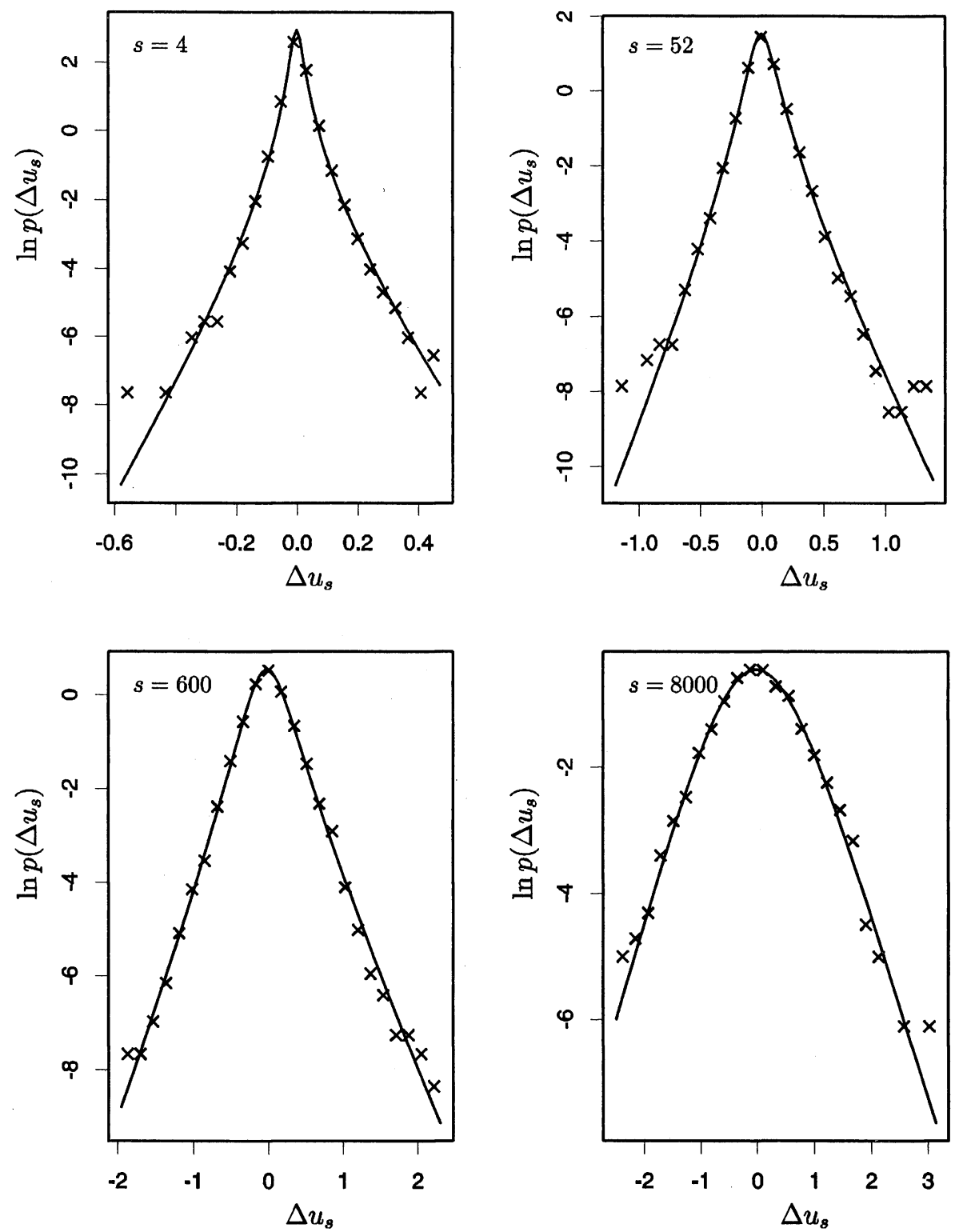

Fig. 2.1. Approximation of the PDF of velocity increments within the class of $N I G$ distributions (solid lines, fitting by maximum likelihood) for data from the atmospheric boundary layer (kindly provided by K. R. Sreenivasan) with $R_{\lambda}=17000$ and time scales $s=4,52,600,8000$ (in units of the finest resolution).

tractable distributions, the normal inverse Gaussian $(N I G)$ distributions. Fig. 2.1 shows, as an example, the $\log$ densities of velocity increments $\Delta u_{s}=u_{t+s}-u_{t}$ measured in the atmospheric boundary layer for various time spans $s$. The solid lines denote the approximation of these densities 
within the class of $N I G$ distributions. $N I G$ distributions fit the empirical densities equally well for all time scales $s$ (for a more detailed verification, see [1]). Furthermore, the subsequent analysis of the observed parameters of the NIG distributions revealed that the PDFs of different data sets with different Taylor-based Reynolds numbers (ranging from $R_{\lambda}=80$ up to $R_{\lambda}=17000$ ) all collapse after applying a scale transformation that is related to one of the parameters of the estimated $N I G$ distributions. As a consequence, the collapse of PDFs immediately resulted in a broader and more general reformulation of the concept of Extended Self-Similarity [6] in terms of a stochastic equivalence class.

The analysis in [1] is to a large extent based on key empirical facts, without providing a theoretical model for the turbulent velocity field. In view of the significance of the derived results, a theoretical basis is clearly asked for. In the present paper we propose a stochastic differential equation framework for modelling the timewise dynamics of the main component of the velocity, that is able to reproduce the observed evolution of the PDF of turbulent velocity increments.

The second point we want to make in this paper is to show that our model is, to a large extent, in accordance with the experimental verification of Kolmogorov's refined similarity hypotheses (K62) [13]. In essence, Kolmogorov refined his 1941 theory [11] by taking into account the strong variability of the local energy dissipation.

The first refined hypothesis states that the PDF of the stochastic variable

$$
V_{r}=\frac{\Delta u(r)}{\left(r \varepsilon_{r}\right)^{1 / 3}}
$$

depends, for $r \ll L$, only on the local Reynolds number $\operatorname{Re}_{r}=r\left(r \varepsilon_{r}\right)^{1 / 3} / \nu$. Here $\Delta u(r)$ denotes a spatial velocity increment at scale $r, \nu$ is the viscosity, $L$ the integral scale and $r \varepsilon_{r}$ is the integrated energy dissipation over a spatial domain of linear size $r$.

The second refined hypothesis states that, for $\operatorname{Re}_{r} \gg 1$, the PDF of $V_{r}$ does not depend on $\mathrm{Re}_{r}$ and is therefore universal. Although, for small $r$, an additional $r$ dependence of the PDF of $V_{r}$ has been observed [22], the validity of several aspects of $\mathrm{K} 62$ has been verified experimentally and by numerical simulation of turbulence [22], [27], [23].

3. Modelling framework. The present section introduces the general type of stochastic process we have in mind for the description of turbulent velocities. The mathematical tools needed for the more detailed specifications made later are outlined in the next section.

We propose to model the dynamics of the main component of the velocity (i.e., the component in the direction of the mean flow) as a stochastic 
integral

$$
u_{t}=\bar{u}+\int_{-\infty}^{t} g(t-s) \mathrm{d} Y_{s}
$$

where $\bar{u}$ is a constant, $g$ is a deterministic kernel and the process $Y$ satisfies a stochastic differential equation

$$
\mathrm{d} Y_{t}=\beta \varepsilon_{t} \mathrm{~d} t+\sqrt{\varepsilon_{t}} \mathrm{~d} B_{t}
$$

where $\beta$ is a constant, $\varepsilon$ denotes a positive stationary process, and $B$ is a Brownian motion. This type of process $Y$ is often encountered in other areas of application. In particular, a modelling framework rather similar to the one proposed here has been demonstrated to allow for a model specification that captures the key stylized features of the time evolution of stock prices and exchange rates and is very tractable analytically and numerically, see [4], [5] and references given there. The aim here is to show that suitable choices of $g$ and $\varepsilon$ can reproduce key stylized features of the timewise behavior of the velocity.

Combining (3.1) and (3.2) we get

$$
u_{t}=\bar{u}+\beta \int_{-\infty}^{t} g(t-s) \varepsilon_{s} \mathrm{~d} s+\int_{-\infty}^{t} g(t-s) \sqrt{\varepsilon_{s}} \mathrm{~d} B_{s} .
$$

In the present context of turbulence we conceive of $\varepsilon$ as expressing the time varying intermittency while $B$ generates independent innovation impulses.

The decomposition of the velocity $u_{t}$ in (3.3) is reminiscent of the Reynolds decomposition [15], with $\int g(t-s) \varepsilon_{s} \mathrm{~d} s$ playing the role of the slowly varying component and $\int g(t-s) \sqrt{\varepsilon_{s}} \mathrm{~d} B_{s}$ reflecting the strongly varying component (with mean zero).

A strength of the modelling framework (3.3) lies in the fact that the intermittency generating term $\varepsilon$ and the function $g$ can, to a large extent, be chosen arbitrarily. In the next section we identify $\varepsilon$ with the local energy dissipation. Therefore, the model (3.3) establishes a framework that derives the model for the velocity field directly from the presumed model for the local energy dissipation. The calculations in Section 5 show that a considerable part of the statistics of the velocity field are independent of the specific choice of the model for the energy dissipation. In particular, both the evolution of the PDF of velocity increments from heavy tails to an approximate Gaussian shape with increasing scale and the statistics related to K62 are predominantly mediated by the structure of (3.3).

$\mathrm{R}$ e $\mathrm{m}$ a $\mathrm{rk}$ 3.1. The framework (3.1) can be generalized to a model for the full velocity vector $u_{t}(\sigma)$ at time $t$ and position $\sigma$

$$
u_{t}(\sigma)=\bar{u}+\int_{-\infty}^{t} \int_{S} g(t-s ;|\rho-\sigma|) \mathrm{d} M_{s}(\mathrm{~d} \rho),
$$


where $M$ is a random measure on $\mathbf{R} \times S, S$ denoting the space of possible locations. For an initial discussion of this more general framework we refer to [3].

4. Mathematical background. This section outlines the mathematical tools we require for the more detailed modelling of the turbulent velocity process $u_{t}$. The basic notions are semimartingales, Lévy processes and quadratic variation. For later purposes we also provide the definitions and basic properties of normal inverse Gaussian distributions and inverse Gaussian distributions. While the former approximates the distribution of temporal velocity increments for all time scales and all amplitudes, the latter will be used to model explicitely the intermittency of the velocity.

The stochastic processes we propose as a model for the turbulent velocity are nowhere differentiable, thus the definition of the energy dissipation as the square of velocity derivatives does not make sense in this context. As an alternative definition of the energy dissipation we propose to use the concept of quadratic variation, as outlined below.

4.1. Semimartingales and quadratic variation. In the language of stochastic analysis the process $u$, as given by (3.3), is - subject to minor regularity conditions on $g$ and $\varepsilon-$ a Brownian semimartingale. Specifically we assume that $\varepsilon$ is càdlàg, i.e., it is a stochastic process whose sample paths at all time points are continuous from the right and have limits from the left. Furthermore, we require that $\varepsilon$ has finite mean and that the deterministic function $g$ is nonnegative, bounded by 1 with $g(0)=1$, and differentiable and square integrable on $[0, \infty)$.

A key result of stochastic analysis states that for any semimartingale $u$, whether Brownian or not, the limit

$$
[u]_{t}=\lim _{n \rightarrow \infty} \sum_{j=1}^{n}\left(u_{t j / n}-u_{t(j-1) / n}\right)^{2}
$$

exists, as a limit in probability. The derived process $[u]$ expresses the cumulative variation exhibited by $u$ and is called the quadratic variation (QV). The monograph [17] is a comprehensive account of basic parts of stochastic analysis. For further properties, see [10].

We may calculate $[u]$ from (3.3) using Itô algebra. Specifically, the stochastic differential of $u$ is

$$
\mathrm{d} u_{t}=a_{t} \mathrm{~d} t+\sqrt{\varepsilon_{t}} \mathrm{~d} B_{t},
$$

where

$$
a_{t}=\beta \varepsilon_{t}+\beta \int_{-\infty}^{t} g^{\prime}(t-s)\left(\varepsilon_{s} \mathrm{~d} s+\sqrt{\varepsilon_{s}} \mathrm{~d} B_{s}\right)
$$

and $A_{t}=\int_{0}^{t} a_{s} \mathrm{~d} s$ is of finite variation. Thus

$$
\left(\mathrm{d} u_{t}\right)^{2}=a_{t}^{2}(\mathrm{~d} t)^{2}+2 a_{t} \sqrt{\varepsilon_{t}} \mathrm{~d} t \mathrm{~d} B_{t}+\varepsilon_{t}\left(\mathrm{~d} B_{t}\right)^{2} .
$$


By Itô algebra $(\mathrm{d} t)^{2}=0, \mathrm{~d} t \mathrm{~d} B_{t}=0$ while $\left(\mathrm{d} B_{t}\right)^{2}=\mathrm{d} t$. All in all, we obtain

$$
\left(\mathrm{d} u_{t}\right)^{2}=\varepsilon_{t} \mathrm{~d} t
$$

and

$$
[u]_{t}=\int_{0}^{t}\left(\mathrm{~d} u_{s}\right)^{2}=\int_{0}^{t} \varepsilon_{s} \mathrm{~d} s .
$$

In the setting of stochastic differential equations of the Brownian semimartingale type the quantity $\left(\mathrm{d} u_{t}\right)^{2} / \mathrm{d} t$ is the natural analogue of the squared first order derivative of the velocity, which in the classical formulation is taken to express the local energy dissipation. Consequently, the quadratic variation $[u]_{t}$ is the stochastic analogue of the integrated energy dissipation and $\varepsilon_{t}$ can be identified with the local energy dissipation.

It is to note that the quadratic variation $[u]_{t}$ is independent of $\beta$, i.e., independent of the second term in (3.3). That term is responsible for the skewness of velocity increments. The skewness of the distribution of $u_{t}-u_{0}$ has a relatively complicated expression, and in this paper we restrict attention to the infinitesimal skewness $\mathbf{E}\left\{\left(\mathrm{d} u_{t}\right)^{3}\right\}$, noting that $\mathbf{E}\left\{\mathrm{d} u_{t}\right\}=0$ due to the stationarity of $u_{t}$. Here, $\mathbf{E}\{\cdot\}$ denotes the expectation.

For simplicity, from now on we assume that the processes $\varepsilon$ and $B$ are independent.

In particular, we then, using (4.2), obtain the following formula for the infinitesimal skewness:

$$
\mathbf{E}\left\{\left(\mathrm{d} u_{t}\right)^{3}\right\}=3 \beta\left[\mathbf{E}\left\{\varepsilon_{0}^{2}\right\}+\int_{0}^{\infty} g^{\prime}(s) \mathbf{E}\left\{\varepsilon_{0} \varepsilon_{s}\right\} \mathrm{d} s\right](\mathrm{d} t)^{2} .
$$

Under the additional simplifying (weak) assumptions

$$
\int_{0}^{\infty}\left|g^{\prime}(t)\right| \mathrm{d} t=1
$$

and

$$
\mathbf{E}\left\{\varepsilon_{0}^{2}\right\}-\mathbf{E}\left\{\varepsilon_{0} \varepsilon_{t}\right\}>0,
$$

and $g$ monotonically decreasing, we finally get

$$
\mathbf{E}\left\{\left(\mathrm{d} u_{t}\right)^{3}\right\}=3 \beta(\mathrm{d} t)^{2} \int_{0}^{\infty}\left|g^{\prime}(s)\right|\left[\mathbf{E}\left\{\varepsilon_{0}^{2}\right\}-\mathbf{E}\left\{\varepsilon_{0} \varepsilon_{s}\right\}\right] \mathrm{d} s>0 .
$$

This result is in accordance with the positive skewness of temporal turbulent velocity increments as follows from the famous $\frac{4}{5}$-law of Kolmogorov [12], invoking Taylor's Frozen Flow Hypothesis [24]. In our stochastic framework (3.3), the positive skewness of temporal velocity increments is directly related to the positive autocorrelation (4.9) of the local energy dissipation.

$\mathrm{R}$ e $\mathrm{m}$ a $\mathrm{r} \mathrm{k}$ 4.1. Kolmogorov's 4/5-law predicts a negative skewness for the distribution of spatial velocity increments $u(x+r)-u(x)$, where $r$ is a spatial distance along the direction of the mean flow. Timewise velocity 
increments $u_{t+s}-u_{t}$, where $s$ is a positive time lag, show a positive skewness [1], [8]. This change of sign of the skewness by going from spatial to temporal statistics can be explained using Taylor's Frozen Flow Hypothesis stating that the temporal variation $u_{t+s}-u_{t}$ at a fixed spatial location can be interpreted as a spatial variation $u(x)-u(x+r)$ at a fixed time by setting $r=U s$, where $U$ denotes the mean velocity. Here it is important to note that $u_{t+s}$ corresponds to $u(x)$ and $u_{t}$ to $u(x+r)$ (for positive $r$ in the direction of the mean flow), which is the correspondence responsible for the change of sign of the skewness.

4.2. Lévy processes and OU processes. Besides the Brownian semimartingales two other basic types of semimartingales are Lévy processes and Ornstein-Uhlenbeck (OU) processes. These are also central to our general modelling approach.

A Lévy process is a stochastic process with càdlàg sample paths and independent and identically distributed increments. The Poisson process and the stable processes (Lévy flights) as well as Brownian motion are of this type. But the class of Lévy processes is much wider than this, the inverse Gaussian and the normal inverse Gaussian Lévy processes being important examples; these are Lévy processes for which the laws of the increments are, respectively, inverse Gaussian and normal inverse Gaussian (for definition and properties of these laws, which are denoted by $I G$ and $N I G$, respectively, see Subsection 4.3 below).

The Lévy processes, other than Brownian motion, enter our modelling framework only indirectly via the concept of OU processes. An OU process is a stationary process $Z$ satisfying a stochastic differential equation of the form

$$
\mathrm{d} Z_{t}=-\lambda Z_{t} \mathrm{~d} t+\mathrm{d} L_{\lambda t},
$$

where $L$ is a Lévy processes, called the background driving Lévy process (BDLP). This equation has a stationary solution for any Lévy process $L$ such that $\mathbf{E}\left\{\ln \left(1+\left|L_{1}\right|\right)\right\}<\infty$. In particular, taking $L$ to be an inverse Gaussian Lévy process we obtain as solution $Z$ the so-called OU-IG process, which will be applied in the sequel, as a model for the intermittency.

OU-IG processes have positive sample paths. Integrated with respect to Brownian motion, they have the property to show a pronounced $N I G$-shape with heavy tails for the increments at small scales. For the large scales, the PDF of increments tends to a Gaussian-like shape. This is the property we want to model for the turbulent velocity.

\subsection{Normal inverse Gaussian and inverse Gaussian distribu-} tions. The normal inverse Gaussian law, with parameters $\alpha, \beta, \mu$, and $\delta$, is the distribution on the real axis $\mathbf{R}$ having probability density function

$$
p(x ; \alpha, \beta, \mu, \delta)=a(\alpha, \beta, \mu, \delta) q\left(\frac{x-\mu}{\delta}\right)^{-1} K_{1}\left\{\delta \alpha q\left(\frac{x-\mu}{\delta}\right)\right\} e^{\beta x},
$$


where $q(x)=\sqrt{1+x^{2}}$ and

$$
a(\alpha, \beta, \mu, \delta)=\pi^{-1} \alpha \exp \left\{\delta \sqrt{\alpha^{2}-\beta^{2}}-\beta \mu\right\}
$$

and where $K_{1}$ is the modified Bessel function of the third kind and index 1 . The domain of variation of the parameters is given by $\mu \in \mathbf{R}, \delta \in \mathbf{R}_{+}$, and $0 \leqslant|\beta|<\alpha$. The distribution is denoted by $N I G(\alpha, \beta, \mu, \delta)$.

The standardized third and fourth order cumulants are

$$
\bar{c}_{3}=\frac{c_{3}}{c_{2}^{3 / 2}}=3 \frac{\rho}{\left(\delta \alpha\left(1-\rho^{2}\right)^{1 / 2}\right)^{1 / 2}}, \quad \bar{c}_{4}=\frac{c_{4}}{c_{2}^{2}}=3 \frac{1+4 \rho^{2}}{\delta \alpha\left(1-\rho^{2}\right)^{1 / 2}},
$$

where $\rho=\beta / \alpha$. We note that the $N I G$ distribution (4.12) has semi-heavy tails; specifically,

$$
p(x ; \alpha, \beta, \mu, \delta) \sim \text { const }|x|^{-3 / 2} \exp \{-\alpha|x|+\beta x\}, \quad x \rightarrow \pm \infty .
$$

NIG shape triangle. For some purposes it is useful, instead of the classical skewness and kurtosis quantities (4.14), to work with the alternative asymmetry and steepness parameters $\chi$ and $\xi$ defined by

$$
\chi=\rho \xi \quad \text { and } \quad \xi=(1+\bar{\gamma})^{-1 / 2}
$$

where $\bar{\gamma}=\delta \sqrt{\alpha^{2}-\beta^{2}}$. Like $\bar{c}_{3}$ and $\bar{c}_{4}$, these parameters are invariant under location-scale changes and the domain of variation for $(\chi, \xi)$ is the normal inverse Gaussian shape triangle

$$
\{(\chi, \xi):-1<\chi<1,0<\xi<1\} .
$$

The distributions with $\chi=0$ are symmetric, and the normal law occurs as limiting case for $(\chi, \xi)$ near to $(0,0)$. Fig. 4.1 gives an impression of the shape of the NIG distributions for various values of $(\chi, \xi)$. The dashed line in Fig. 4.1 corresponds to $\rho=0.1$ and represents the approximate location of the PDF of temporal turbulent velocity increments, as reported in [1].

As discussed in the papers cited in [1], the class of $N I G$ distributions and processes have been found to provide accurate modelling of a great variety of empirical findings in the physical sciences and in financial econometrics.

As a second infinitely divisible distribution we need the inverse Gaussian distribution $(I G)$. This distribution will be used to model the intermittency of the velocity field. The inverse Gaussian law, with parameters $\delta$ and $\gamma$, is the distribution on the positive real axis $\mathbf{R}_{+}$having probability density function

$$
p(x ; \delta, \gamma)=\frac{\delta}{\sqrt{2 \pi}} e^{\delta \gamma} x^{-3 / 2} \exp \left\{-\frac{\delta^{2} x^{-1}+\gamma^{2} x}{2}\right\},
$$

where the parameters $\delta$ and $\gamma$ satisfy $\delta>0$ and $\gamma \geqslant 0$. 


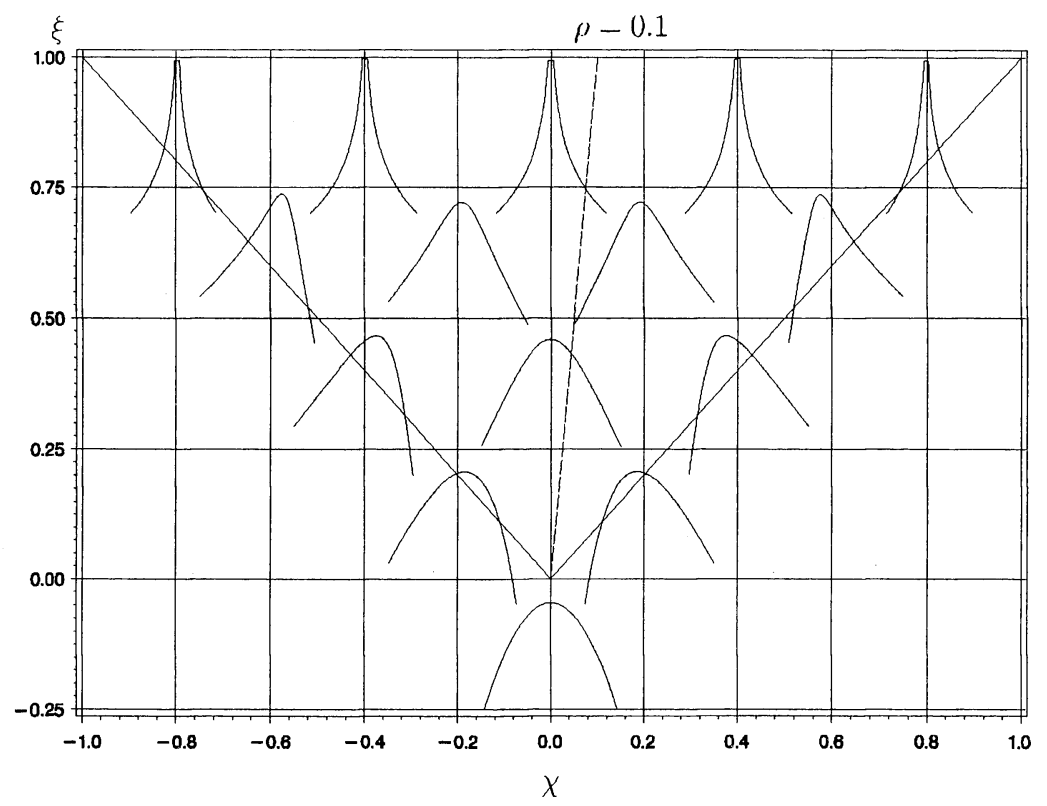

Fig. 4.1. The shape triangle of the $N I G$ distributions with the log density functions of the standardized distributions, i.e., with mean 0 and variance 1 , corresponding to the values $(\chi, \xi)=( \pm 0.8,0.999),( \pm 0.4,0.999),(0.0,0.999),( \pm 0.6,0.75),( \pm 0.2,0.75),( \pm 0.4,0.5)$, $(0.0,0.5),( \pm 0.2,0.25)$, and $(0.0,0.0)$. The coordinate system of the log densities is placed at the corresponding value of $(\chi, \xi)$. Furthermore, the line corresponding to $\rho=0.1$, i.e., $\chi=0.1 \xi$, is shown.

5. Temporal model for the turbulent velocity field. Section 3 introduced the modelling framework for the turbulent velocity in its full generality. Here, we focus on two specific properties of the turbulent velocity, namely the evolution of the PDF of velocity increments across time scales, and statistics related to $\mathrm{K} 62$.

For mathematical convenience we neglect the skewness of the velocity field, setting $\beta=0$ in (3.3). The skewness of the velocity field is not essential for the evolution of the PDF of velocity increments from heavy tails at small scales to an approximate Gaussian shape at large scales. We also expect that neglecting the skewness of the velocity field does not alter the basic statistical properties of the Kolmogorov variable (2.1), in particular its conditional distributions. A more detailed discussion of the influence of the skewness term will be given elsewhere.

5.1. Evolution of the PDF of velocity increments. We discuss the PDF of velocity increments $u_{t}-u_{0}$, where $t>0$, in terms of cumulants. In our nonskewed set-up, i.e., (3.3) with $\beta=0$, the third order cumulant is zero for all scales $t$. Therefore, the fourth order cumulant is the first order that distinguishes between a Gaussian like shape for the large scales 
and heavy tails for small scales. Without specifying the function $g$ and the local energy dissipation process $\varepsilon$ in detail, the large scale limit of $u_{t}-u_{0}$ approaches a Gaussian shape. Note however that under model (3.3) the limit law of $u_{t}-u_{0}$ for $t \rightarrow \infty$ can never be Gaussian unless the intermittency process $\varepsilon$ is deterministic. In general, $u_{t}-u_{0}$ will tend in law to a random variable of the form $v_{0}-u_{0}$, where $v_{0}$ is an independent copy of $u_{0}$, and the law of $v_{0}-u_{0}$ is mixed Gaussian when $\varepsilon$ is independent of the Brownian motion $B$. In addition, we are able to show analytically, for specific choices of $g$ and $\varepsilon_{t}$, that the small scale limit has pronounced heavy tails.

We shall denote the $m$ th order cumulant of an arbitrary random variable $u$ by $c_{m}(u)$ and write the cumulant function of $u$ as

$$
\mathrm{C}\{\zeta \ddagger u\}=\ln \mathbf{E}\left\{e^{i \zeta u}\right\} .
$$

Furthermore, for any positive random variable $X$ we define the cumulant function $\overline{\mathrm{K}}$ of $X$ by

$$
\overline{\mathrm{K}}\{\theta \ddagger X\}=\ln \mathbf{E}\left\{e^{-\theta X}\right\} .
$$

To get some insight into the statistical properties of the stationary increments $u_{t}-u_{0}$, we first calculate the cumulant function. We have

$$
u_{t}-u_{0}=\int_{-\infty}^{t}\left[g(t-s)-\mathbf{1}_{(-\infty, 0]}(s) g(-s)\right] \sqrt{\varepsilon_{s}} \mathrm{~d} B_{s},
$$

where $\mathbf{1}_{I}$ denotes the indicator function on an interval $I$. Since, conditionally on $\varepsilon$, the process $u$ is Gaussian, we get for the cumulant function of $u_{t}-u_{0}$ the form

$$
\mathrm{C}\left\{\zeta \ddagger u_{t}-u_{0}\right\}=\overline{\mathrm{K}}\left\{\frac{1}{2} \zeta^{2} \ddagger Q(t)\right\},
$$

where

$$
Q(t)=c_{2}\left(u_{t}-u_{0} \mid \varepsilon\right)=\int_{-\infty}^{t}\left[g(t-s)-\mathbf{1}_{(-\infty, 0]}(s) g(-s)\right]^{2} \varepsilon_{s} \mathrm{~d} s
$$

is the conditional variance of $u_{t}-u_{0}$ given $\varepsilon$.

Differentiating the cumulant function (5.4) gives

$$
c_{2}\left(u_{t}-u_{0}\right)=\mathbf{E}\{Q(t)\}=c_{1}\left(\varepsilon_{0}\right) G(t),
$$

where

$$
G(t)=\int_{-\infty}^{t}\left[g(t-s)-\mathbf{1}_{(-\infty, 0]}(s) g(-s)\right]^{2} \mathrm{~d} s .
$$

Furthermore,

$$
\frac{1}{3} c_{4}\left(u_{t}-u_{0}\right)=c_{2}(Q(t))=c_{2}\left(\varepsilon_{0}\right)\langle g, \tau\rangle(t),
$$


where

$$
\begin{aligned}
\langle g, \tau\rangle(t)=\int_{-\infty}^{t} \int_{-\infty}^{t}[ & \left(g(t-s)-\mathbf{1}_{(-\infty, 0]}(s) g(-s)\right) \\
& \left.\times\left(g\left(t-s^{\prime}\right)-\mathbf{1}_{(-\infty, 0]}\left(s^{\prime}\right) g\left(-s^{\prime}\right)\right)\right]^{2} \\
& \times \tau\left(\left|s-s^{\prime}\right|\right) \mathrm{d} s \mathrm{~d} s^{\prime}
\end{aligned}
$$

and where $\tau$ is the autocorrelation function of $\varepsilon$.

It follows that

$$
\bar{c}_{4}\left(u_{t}-u_{0}\right)=\frac{c_{4}\left(u_{t}-u_{0}\right)}{c_{2}\left(u_{t}-u_{0}\right)^{2}}=3 \frac{c_{2}\left(\varepsilon_{0}\right)}{c_{1}\left(\varepsilon_{0}\right)^{2}} D(t),
$$

where

$$
D(t)=\frac{\langle g, \tau\rangle(t)}{G(t)^{2}} .
$$

For the small scale limit we get from (5.7), (5.9), and (5.11)

$$
\lim _{t \rightarrow 0} D(t)=\tau(0)=1 \text {. }
$$

For the large scale limit we get

$$
\lim _{t \rightarrow \infty} D(t)=\int_{0}^{\infty} \int_{0}^{\infty} \bar{g}^{2}(s) \bar{g}^{2}\left(s^{\prime}\right) \tau\left(\left|s-s^{\prime}\right|\right) \mathrm{d} s \mathrm{~d} s^{\prime} \leqslant 1,
$$

where

$$
\bar{g}^{2}(t)=\frac{g^{2}(t)}{\int_{0}^{\infty} g^{2}(s) \mathrm{d} s} .
$$

Consequently, $\bar{c}_{4}\left(u_{t}-u_{0}\right)$ will be small for large $t$ if either $c_{2}\left(\varepsilon_{0}\right) / c_{1}\left(\varepsilon_{0}\right)^{2}$ is small or the interplay between the decrease of $g$ and $\tau$ results in $\lim _{t \rightarrow \infty} D(t) \ll 1$. In both cases we may expect the law of $u_{t}-u_{0}$ to be close to Gaussian for large $t$ (without being strictly Gaussian).

In the case where $c_{2}\left(\varepsilon_{0}\right) / c_{1}\left(\varepsilon_{0}\right)^{2}$ is not small, but $g$ or $\tau$ decreases fast enough for $\lim _{t \rightarrow \infty} D(t) \ll c_{1}\left(\varepsilon_{0}\right)^{2} /\left(3 c_{2}\left(\varepsilon_{0}\right)\right)$ we may expect our model (3.3) to show the evolution of the PDF of temporal velocity increments from heavy tails at small scales to an approximate Gaussian shape at large scales.

5.2. Statistics of the Kolmogorov variable. We now turn to the discussion of the statistics of the Kolmogorov variable $V$ of (2.1) within our stochastic framework (3.3) with $\beta=0$. In particular, we show that the Kolmogorov variable $V$ can be represented as the product of two independent variables, namely a standard normal random variable and a process that completely contains the dependence of $V$ on the integrated energy dissipation. Based on this decomposition, some analytical results concerning the conditional PDF of $V$ for the small and large scale limits can be derived. 
Following the discussion in Subsection 4.1 we replace the integrated energy dissipation in (2.1) by the quadratic variation and define the stochastic analogue of the classical Kolmogorov variable $V$ [13] as

$$
V_{t}=\frac{u_{t}-u_{0}}{\left\{\bar{u}[u]_{t}\right\}^{1 / 3}}
$$

The introduction of the mean velocity $\bar{u}$ turns $V_{t}$ into a nondimensional stochastic process.

To reveal the basic statistical properties of the process $V_{t}$ we note that (5.15) may be rewritten as

$$
V_{t}=\frac{u_{t}-u_{0}}{Q(t)^{1 / 2}} \frac{Q(t)^{1 / 2}}{\left\{\bar{u}[u]_{t}\right\}^{1 / 3}}=U R_{t}
$$

where

$$
U=\frac{u_{t}-u_{0}}{Q(t)^{1 / 2}} \quad \text { and } \quad R_{t}=\frac{Q(t)^{1 / 2}}{\left\{\bar{u}[u]_{t}\right\}^{1 / 3}} .
$$

The variable $U$ is a standard normal random variable independent of $R_{t}$. The dependence of $V_{t}$ on $[u]_{t}$ - or, equivalently, $\varepsilon_{t}$ - is thus completely contained in the process $R_{t}$.

To proceed further, we specify the function $g$ to be of the form

$$
g(t)=e^{-\psi t} \text {. }
$$

We gain some insight into the properties of the process $R_{t}$ for $t \rightarrow 0$ noting the decomposition of the conditional variance of velocity increments

$$
Q(t)=\left[1-e^{-\psi t}\right]^{2} \int_{-\infty}^{0} e^{2 \psi s} \varepsilon_{s} \mathrm{~d} s+\left[\int_{0}^{t} e^{-2 \psi(t-s)} \varepsilon_{s} \mathrm{~d} s-[u]_{t}\right]+[u]_{t} .
$$

Focusing on the first term on the right-hand side of (5.19) we get in leading order for $t \rightarrow 0$

$$
\mathbf{E}\left\{\left[1-e^{-\psi t}\right]^{2} \int_{-\infty}^{0} e^{2 \psi s} \varepsilon_{s} \mathrm{~d} s\right\}=c_{1}\left(\varepsilon_{0}\right)[2 \psi]^{-1}\left[1-e^{-\psi t}\right]^{2} \sim \frac{c_{1}\left(\varepsilon_{0}\right) \psi}{2} t^{2} .
$$

For the second term in (5.19) we have, by (4.6)

$$
\int_{0}^{t} e^{-2 \psi(t-s)} \varepsilon_{s} \mathrm{~d} s-[u]_{t}=-\int_{0}^{t}\left[1-e^{-2 \psi(t-s)}\right] \varepsilon_{s} \mathrm{~d} s
$$

and in the limit $t \rightarrow 0$, to leading order

$$
\mathbf{E}\left\{\int_{0}^{t}\left[1-e^{-2 \psi(t-s)}\right] \varepsilon_{s} \mathrm{~d} s\right\}=c_{1}\left(\varepsilon_{0}\right)\left[t-(2 \psi)^{-1}\left(1-e^{-2 \psi t}\right)\right] \sim 2 c_{1}\left(\varepsilon_{0}\right) \psi t^{2} .
$$

Since the first term in (5.19) is strictly positive and the second one is strictly negative we conclude that they are both predominantly of order $t^{2}$ for small $t$. 
Therefore, since the mean of $[u]_{t}$ is linear in $t$, we conclude that the quadratic variation dominates in (5.19) for small $t$ and consequently

$$
V_{t} \sim U[u]_{t}^{1 / 6}
$$

The small scale dependence of $V_{t}$ on the integrated energy dissipation is in conformity with the corresponding result for the turbulent velocity field that follows from kinematic considerations at scales smaller than dissipation scales.

We can also draw a conclusion for the large scale limit $t \rightarrow \infty$. If we assume the intermittency process $\varepsilon_{t}$ to be ergodic, we get $[u]_{t} \sim t c_{1}\left(\varepsilon_{0}\right)$. Furthermore, since

$$
\mathbf{E}\{Q(t)\}=c_{1}\left(\varepsilon_{0}\right) \psi^{-1}\left[1-e^{-\psi t}\right]
$$

we get for $t \rightarrow \infty$

$$
\mathbf{E}\left\{\left|V_{t}\right|\right\} \sim t^{-1 / 3}
$$

The behavior $\mathbf{E}\left\{\left|V_{t}\right|\right\} \propto t^{-0.4}$ is reported for high Reynolds number atmospheric data in [22]. In the analysis of [22] the range of $t$, where the exponent 0.4 holds, is small. For larger $t$ an exponent of $\frac{1}{3}$ seems to better fit the data [22].

The small scale limit (5.23) and the large scale limit (5.25) are both in accordance with the corresponding experimental results. For the time being we are not able to analytically treat the case of moderate $t$ which is the most interesting in view of K62. For these scales we have to refer to the simulation in the next section.

6. Simulation. The analytical results in the last section mainly concern the statistics of velocity increments and the statistics of the Kolmogorov variable for the small and large scale limits. The corresponding results for moderate scales are only accessible through numerical simulation.

For the simulations we use a discretized version of the nonskewed model (3.3) with $\beta=0$. For the weight function we set

$$
g(t)=e^{-\psi t} \mathbf{1}_{[0, T]},
$$

where $\psi$ and $T$ are positive numbers. The introduction of $T$ associates a finite decorrelation time to the velocity field $u$. We further specify the process $\varepsilon$ as a truncated OU-IG process, i.e.,

$$
\varepsilon_{t}=\int_{t-\bar{T}}^{t} e^{-\lambda(t-s)} \mathrm{d} L_{\lambda s}
$$

where $L$ is an $I G(\delta, \gamma)$-Lévy process. The assumption (6.2) coincides for $\bar{T} \rightarrow \infty$ with the definition of an ordinary OU- $I G$ process.

The values for the parameters of the simulation of $u$ are $\bar{u}=1, \lambda=1$, $\bar{T}=100, \psi=0.1, \delta=1, \gamma=1$, and $T=40$ and we discretized all 
stochastic integrals with a finite step size $\Delta t=1$. Hence we simulated, for $t=0,1, \ldots, N$ with $N=2 \cdot 10^{6}$,

$$
\varepsilon_{t}=\sum_{j=t-\bar{T}}^{t-1} e^{-\lambda(t-j)}\left(L_{j+1}-L_{j}\right)
$$

and

$$
u_{t}=\sum_{j=t-T}^{t-1} e^{-\psi(t-j)} \sqrt{\varepsilon_{j}}\left(B_{j+1}-B_{j}\right) .
$$

For the quadratic variation we used the approximation

$$
[u]_{t}=\sum_{j=0}^{t-1}\left(u_{j+1}-u_{j}\right)^{2}
$$

which coincides with the usual definition of the energy dissipation for the temporal resolution $\Delta t=1$.
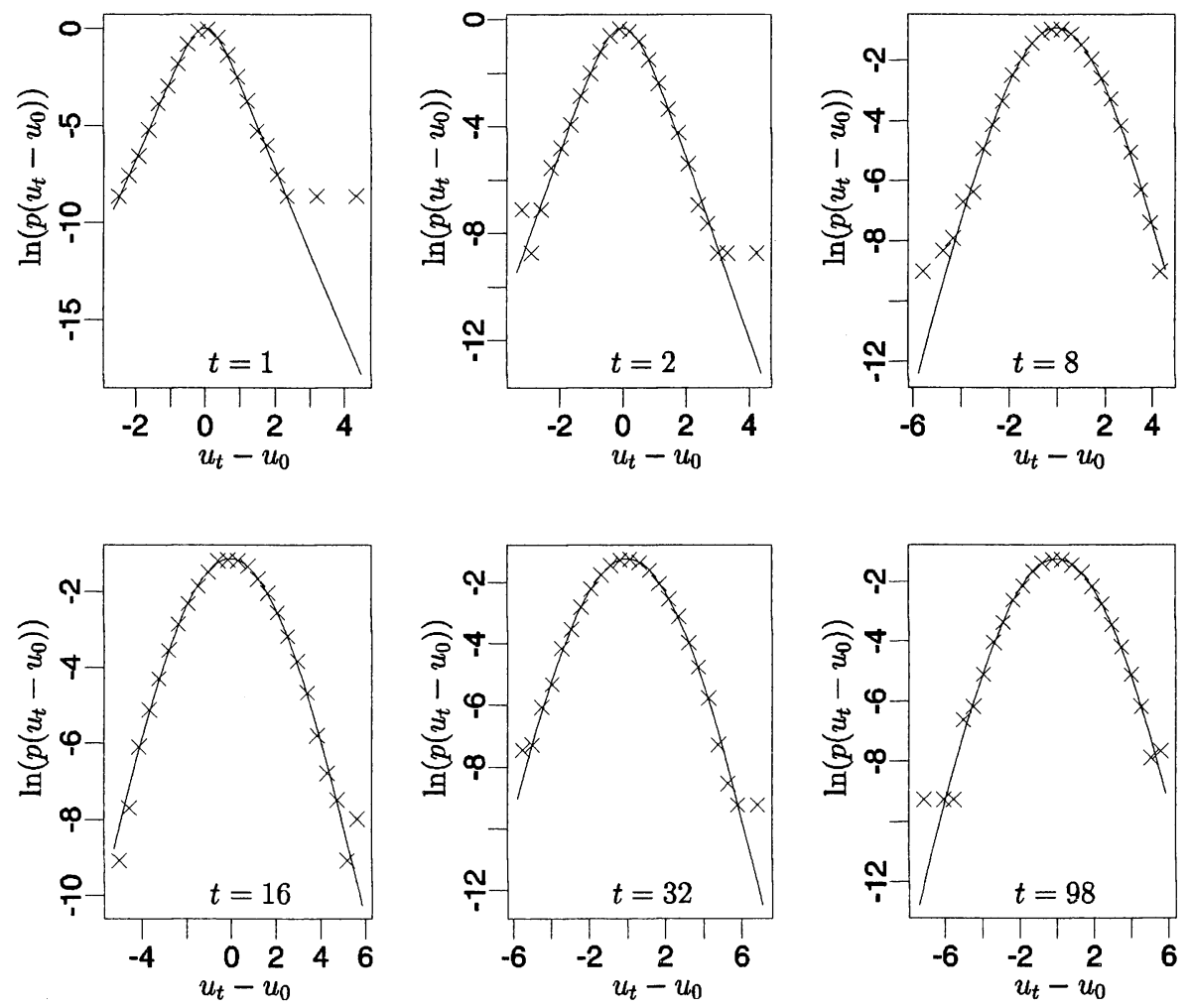

Fig. 6.1. Logarithm of the probability densities of the simulated increments $u_{t}-u_{0}$ (arbitrary units) with $t=1,2,8,16,32,98$ (in units of the finite step size $\Delta t$ ). The solid lines denote the approximation within the class of $N I G$ distributions (fitting by maximum likelihood). 


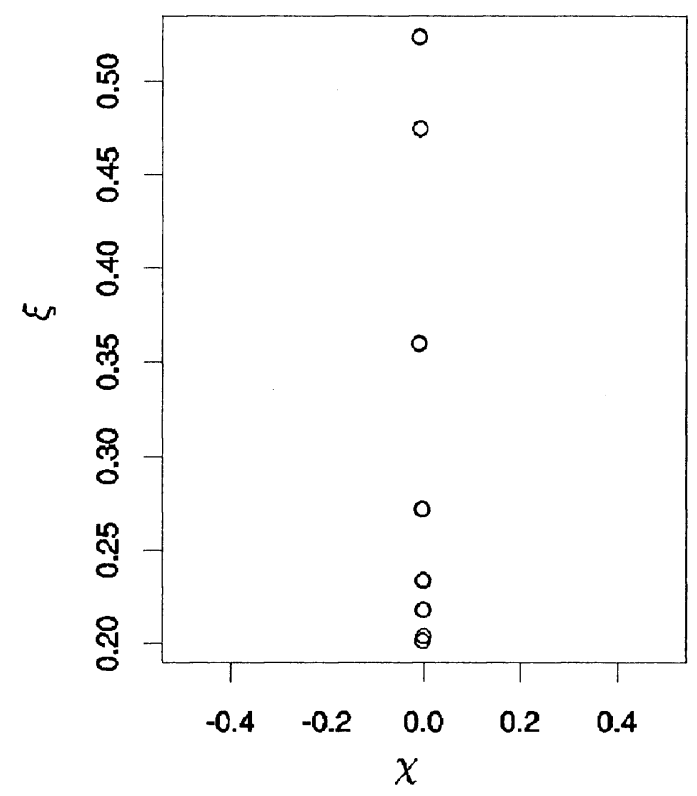

Fig. 6.2. $\quad N I G$ shapes for the densities of the simulated increments $u_{t}-u_{0}$, with $t=1,2,4,8,16,32,64,98$ (from top to bottom, in units of the finite step size $\Delta t$ ).
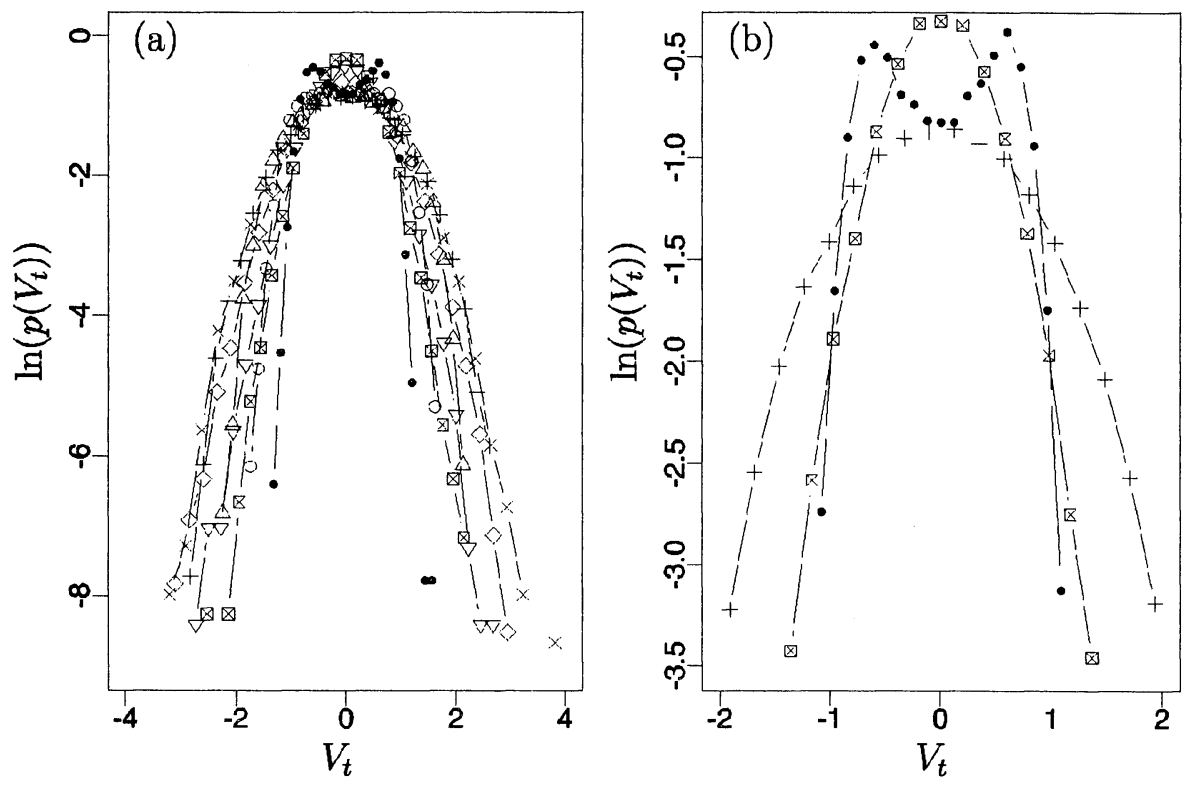

Fig. 6.3. (a) Logarithm of the simulated unconditional densities $p\left(V_{t}\right)$ of the Kolmogorov variable $V_{t}$ for $t=1(\bullet), t=2(\circ), t=4(\triangle), t=8(+), t=16(\times), t=32(\diamond)$, $t=64(\nabla)$, and $t=98(\otimes)$ (in units of the finite step size $\Delta t$ ). (b) Amplification of the relevant part of (a) for $t=1(\bullet), t=8(+)$, and $t=98(\otimes)$ (in units of the finite step size $\Delta t)$. 


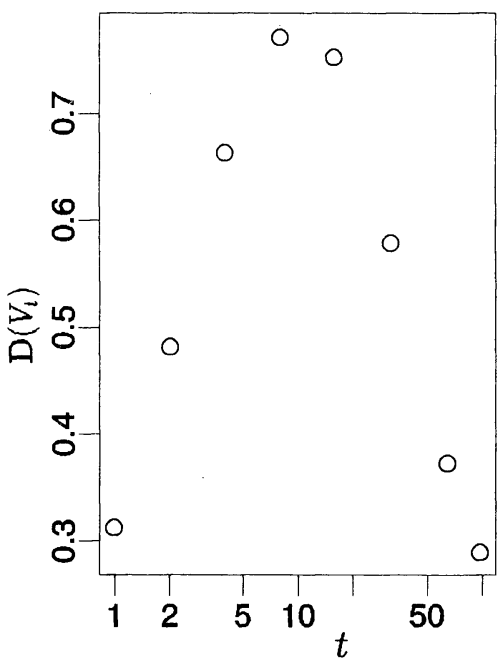

Fig. 6.4. Estimated variance $\mathbf{D}\left(V_{t}\right)$ of the simulated Kolmogorov variable $V_{t}$ with $t=1,2,4,8,16,32,64,98$ (in units of the finite step size $\Delta t$ ).

Fig. 6.1 shows the evolution of the probability densities of the simulated increments $u_{t}-u_{0}$ for various scales $t$. We clearly observe heavy tails for the small scales and an approximately Gaussian shape for the large scales. The solid lines denote the approximation of the densities within the class of $N I G$ distributions. The densities of $u_{t}-u_{0}$ qualitatively display the empirical findings about the evolution across time scales of turbulent velocity increments reported in [1].

We further substantiate the scale dependence in Fig. 6.2 which shows the NIG shapes for the densities as displayed in Fig. 6.1. The parameter $\chi$ is zero for all scales reflecting the symmetry of the densities. The steepness parameter $\xi$ decreases with increasing scale. Noting the expression $\xi=[1+$ $\left.3 / \bar{c}_{4}\right]^{-1 / 2}$ for symmetric $N I G$ distributions, Fig. 6.2 visualizes the evolution from heavy tails (large $\xi$ ) to an approximately Gaussian shape (in the limit $(\xi, \chi) \rightarrow(0,0))$. These findings are very similar to the corresponding results for the turbulent velocity field as reported in [1] (see also Fig. 4.1).

We now turn to the investigation of the Kolmogorov variable $V_{t}$. Fig. 6.3 shows the unconditional densities of $V_{t}$. We first note that the unconditional densities at moderate and large scales are approximately Gaussian, in accordance with the findings in [23], [22], [27], [9]. For not too large scales, the densities collapse for small amplitudes while for large amplitudes, the densities are scale dependent. For the very small scales, a bimodal distribution is observed. The bimodality is related to the heavy tails of the PDF of velocity increments at small scales (in this connection see the mathematical study [14]). 
(a)

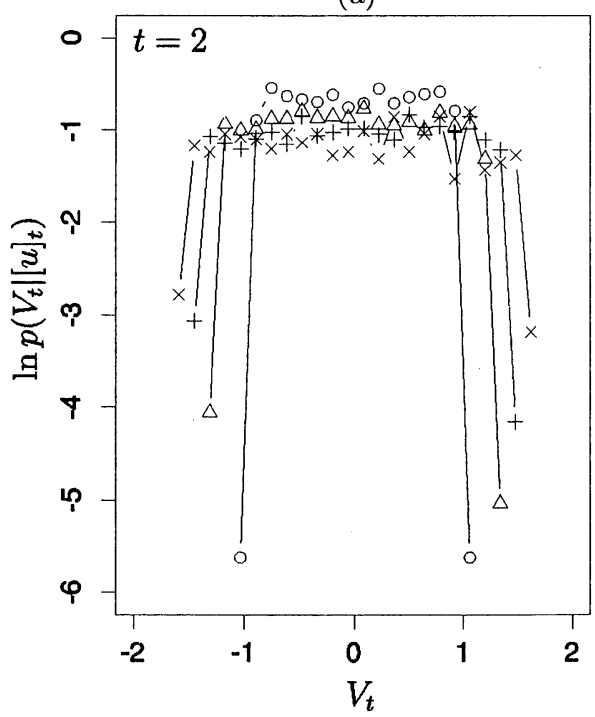

(b)

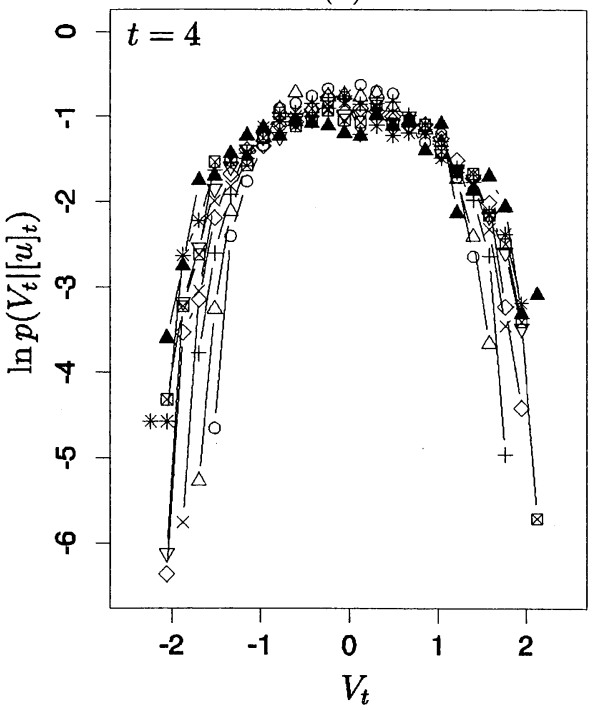

(c)

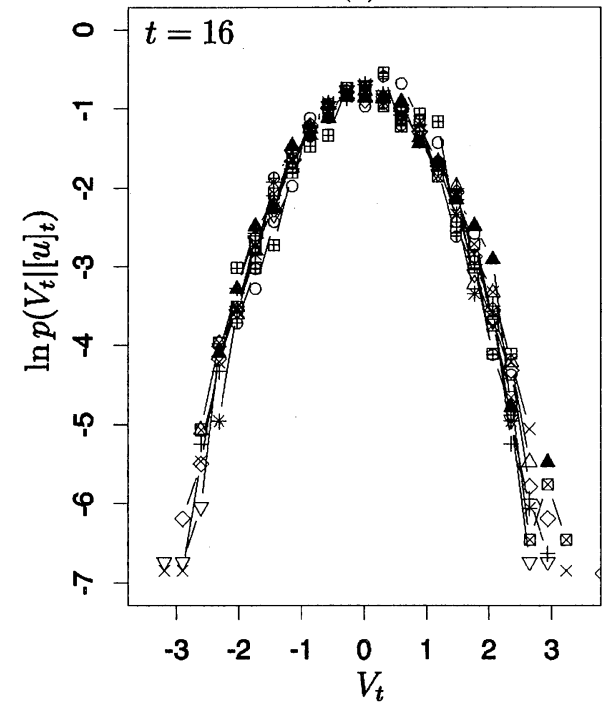

Fig. 6.5. Logarithm of the conditional densities $p\left(V_{t} \mid[u]_{t}\right)$ of the simulated Kolmogorov variable $V_{t}$ :

(a) for $t=2$ (in units of the finite step size $\Delta t$ ) with $[u]_{t}^{1 / 3}=0.45(\mathrm{o}),[u]_{t}^{1 / 3}=0.77$ $(\triangle),[u]_{t}^{1 / 3}=0.99(+)$, and $[u]_{t}^{1 / 3}=1.20(\times)$ (in arbitrary units);

(b) for $t=4$ (in units of the finite step size $\Delta t$ ) with $[u]_{t}^{1 / 3}=0.55(\circ),[u]_{t}^{1 / 3}=0.66$ $(\triangle),[u]_{t}^{1 / 3}=0.76(+),[u]_{t}^{1 / 3}=0.86(\times),[u]_{t}^{1 / 3}=0.96(\diamond),[u]_{t}^{1 / 3}=1.07(\nabla),[u]_{t}^{1 / 3}=1.17$ $(\otimes),[u]_{t}^{1 / 3}=1.27(*)$, and $[u]_{t}^{1 / 3}=1.38(\Delta)$ (in arbitrary units);

(c) for $t=16$ (in units of the finite step size $\Delta t$ ) with $[u]_{t}^{1 / 3}=0.98(\circ),[u]_{t}^{1 / 3}=$ $1.16(\Delta),[u]_{t}^{1 / 3}=1.26(+),[u]_{t}^{1 / 3}=1.35(\times),[u]_{t}^{1 / 3}=1.44(\diamond),[u]_{t}^{1 / 3}=1.53(\nabla)$, $[u]_{t}^{1 / 3}=1.63(\otimes),[u]_{t}^{1 / 3}=1.72(*),[u]_{t}^{1 / 3}=1.81(\mathbf{\Lambda}),[u]_{t}^{1 / 3}=1.9(\oplus)$, and $[u]_{t}^{1 / 3}=2.0$ $(\boxplus)$ (in arbitrary units). 
Comparable results are reported in [27]. The authors discuss the evolution of the second order empirical moments of $V_{t}$ across scales, showing an increase with increasing scale, reaching a plateau at intermediate scales and finally a decrease with further increasing scale. The same behavior holds for the simulation of our model. Fig. 6.4 shows the second order moments of $V_{t}$ as a function of scale $t$.

Fig. 6.5 shows the conditional densities $p\left(V_{t} \mid[u]_{t}\right)$ for various scales $t$ and various values of $[u]_{t}^{1 / 3}$. For small $t$, the conditional densities strongly depend on $[u]_{t}$. With decreasing values of $[u]_{t}^{1 / 3}$, the dependence gets smaller and for large enough $t(t \approx 16$ in our simulation), the conditional densities do not depend on $[u]_{t}$. This independence also holds for the larger scales $16 \leqslant t<\bar{T}$ (not shown here). These findings agree well with results reported for the turbulent velocity field ([23], [22], and [27]) and embody the gist of $\mathrm{K} 62$.

7. Conclusion. Summarizing the main results, we state that our proposed semimartingale framework allows modelling in conformity with the observed evolution of the PDF of temporal velocity increments across time scales and with the experimental verification of Kolmogorov's refined similarity hypotheses. The relation between general stochastic processes and K62 is also discussed in [23]. There fractional Brownian motion ( $\mathrm{fBm}$ ) is proposed as a stochastic process that displays the main properties of K62. However, the use of $\mathrm{fBm}$ there is accompanied with a mathematical inconsistency, connected to the fact that for $\mathrm{fBm}$ (except Brownian motion itself) the quadratic variation is either identically 0 or $\infty$. (This, incidentally, implies that $\mathrm{fBm}$ is not a semimartingale.) Furthermore, fBm is a nonstationary Gaussian process and does not capture the heavy tails for the PDF of velocity increments at small time scales. Thus, to our knowledge, the model (3.3) seems to be the first approach to the turbulent velocity field that comprises both, the evolution of the density of temporal velocity increments across time scales and the statistics of the Kolmogorov variable $V$.

For the simulation we restricted to a very simple form of the intermittency term $\varepsilon_{t}$ as an OU- $I G$ process which is easy to implement but not realistic for the turbulent energy dissipation field. A realistic approach would be to use a more advanced model for the energy dissipation. In particular, we think of Lévy based models that allow one to control explicitly the correlation structure of the energy dissipation field (see [1], [19], [20], and [18]). Controlling the correlation structure of the energy dissipation seems to enable to model the evolution of the density of velocity increments in a way that displays the detailed behavior reported in [1]. A detailed discussion of this will be given elsewhere.

The fact that using an OU- $I G$ process for $\varepsilon_{t}$ works so surprisingly well indicates that models of the form (3.3) are the appropriate framework in 
the turbulence context. In particular, the calculations in Subsection 4.1 and Section 5 show that main parts of the turbulence statistics can be reproduced without specifying the intermittency term $\varepsilon$ and the weight function $g$. In this respect, only a more detailed modelling of the correlation structure of the intermittency can narrow these degrees of freedom.

\section{REFERENCES}

1. Barndorff-Nielsen O.E., Blaesild P., Schmiegel J. A parsimonious and universal description of turbulent velocity increments. - European Phys. J. B, 2004, v. 41, № 3, p. 345-363.

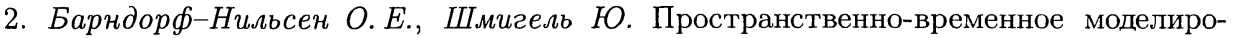
вание, основанное на процессах Леви, и его приложения к турбулентности. Успехи матем. наук, 2004, т. 59, № 1, с. 63-90.

3. Barndorff-Nielsen O.E., Schmiegel J. Ambit processes; with applications to turbulence and tumour growth. - Proceedings of the Abel symposium (Oslo, 2005). Berlin: Springer-Verlag (to appear).

4. Barndorff-Nielsen O. E., Shephard N. Non-Gaussian Ornstein-Uhlenbeck-based models and some of their uses in financial economics. - J. Roy Statist. Soc. Ser. B, 2001, v. 63 , № 2 , p. 167-241.

5. Barndorff-Nielsen O.E., Shephard N. Continuous Time Approach to Financial Volatility. Cambridge: Cambridge Univ. Press (to appear.)

6. Benzi R., Ciliberto S., Tripiccione R., Baudet C., Massaioli F., Succi S. Extended self-similarity in turbulent flows. - Phys. Rev. E, 1993, v. 48, p. R29-R32.

7. Castaing B., Gagne Y., Hopfinger E. J. Velocity probability density functions of high Reynolds number turbulence. - Phys. D, 1990, v. 46, p. 177-200.

8. Фриш У. Турбулентность. Наследие А. Н. Колмогорова. М.: УРСС, 1998, 346 с.

9. Hosokawa I., Van Atta C. W., Thoroddsen S. T. Experimental study of the Kolmogorov refined similarity variable. - Fluid Dyn. Res., 1994, v. 13, p. 329-333.

10. Jacod J., Shiryaev A.N. Limit Theorems for Stochastic Processes. Berlin: SpringerVerlag, 2003, $661 \mathrm{p}$.

11. Колмогоров $A$.Н. Локальная структура турбулентности в несжимаемой вязкой жидкости при очень больших числах Рейнольдса. - Докл. АН СССР, 1941, т. 30, № 4, c. 299-303.

12. Колмогоров А.Н. Рассеяние энергии при локально изотропной турбулентности. - Докл. АН СССР, 1941, т. 32, № 1, с. 19-21.

13. Колмогоров $A . H$. Уточнение представлений о локальной структуре турбулентности в несжимаемой вязкой жидкости при больших числах Рейнольдса. Mécanique de la turbulence, Actes du Colloque International du CNRS (Marseille, 1961). Paris: Éditions du CNRS, 1962, p. 447-458; А. Н. Колмогоров. Избр. труды. Математика и механика. М.: Наука, 1985, с. 348-352.

14. Logan B.F., Mallows C.L., Rice S.O., Shepp L.A. Limit distributions of selfnormalized sums. - Ann. Probab., 1973, v. 1, p. 788-809.

15. Монин А.С., Яглом А.М. Статистическая гидромеханика, ч. 1, 2. М.: Наука, $1965,1967,639$ c., 720 c.

16. Obukhov A. M. Some specific features of atmospheric turbulence. - J. Fluid Mech., 1962 , v. 13 , p. $77-81$.

17. Protter P. E. Stochastic Integration and Differential Equations. Berlin: SpringerVerlag, 2004, $415 \mathrm{p}$.

18. Schmiegel J. Self-scaling of turbulent energy dissipation correlators. - Phys. Lett. A, 2005 , v. 337 , p. $342-353$.

19. Schmiegel J., Barndorff-Nielsen O.E., Eggers H. C. A class of spatio-temporal and causal stochastic processes, with applications to multiscaling and multifractality. South African J. Sci., 2006, v. 101, p. 513-519. 
20. Schmiegel J., Cleve J., Eggers H.C., Pearson B. R., Greiner M. Stochastic energycascade model for $(1+1)$-dimensional fully developed turbulence. - Phys. Lett. A, 2004 , v. 320 , p. $247-253$.

21. Staicu A., van de Water $W$. Small scale velocity jumps in shear turbulence. - Phys. Rev. Lett., 2003, v. 90, 094501.

22. Stolovitzky G., Kailasnath P., Sreenivasan K.R. Kolmogorov's refined similarity hypothesis. - Phys. Rev. Lett., 1992, v. 69, p. 1178-1181.

23. Stolovitzky G., Sreenivasan K.R. Kolmogorov's refined similarity hypotheses for turbulence and general stochastic processes. - Rev. Modern Phys., 1994, v. 66, p. 229-239.

24. Taylor G. I. The spectrum of turbulence. - Proc. Roy. Soc. London Ser. A, 1938, v. 164 , p. $476-490$.

25. van de Water W. Anomalous scaling and anisotropy in turbulence. - Phys. B, 1996, v. 228 , p. $185-191$.

26. Vincent A., Meneguzzi $M$. The spatial structure and statistical properties of homogeneous turbulence. - J. Fluid Mech., 1991, v. 225, p. 1-20.

27. Zhu Y., Antonia R. A., Hosokawa I. Refined similarity hypotheses for turbulent velocity and temperature fields. - Phys. Fluids, 1995, v. 7, p. 1637-1648.

Поступила в редакцию

1.II.2006 